\title{
A COUNTEREXAMPLE OF GEVREY CLASS TO THE UNIQUENESS OF THE CAUCHY PROBLEM
}

\section{LARS HÖRMANDER}

We shall here give a special case of a counterexample due to Alinhac and Baouendi $[1,2]$ which is so explicit that we can show that it belongs to a DenjoyCarleman class contained in every Gevrey class strictly larger than the analytic one. The example of $[1,2]$ is the key to an example of nonuniqueness for the analytic nonlinear Cauchy problem due to Métivier [4], which we also recall adding such strong regularity properties. This may be of some interest since there exist uniqueness theorems which are valid for all Gevrey classes close to the analytic class but not for every Gevrey class (see Lerner [3]).

Theorem 1. Let $\left(L_{\nu}\right)$ be a positive increasing sequence such that

$$
\sum_{1}^{\infty} \frac{1}{\nu \sqrt{L_{\nu}}}<\infty
$$

Then one can find $u$ and $a$ in $C^{\infty}(\Omega), \Omega=\left\{(t, x, y) \in \mathbf{R}^{3} ;|y|<1\right\}$, such that

$$
\begin{gathered}
\left(D_{t}^{2}-2 D_{x} D_{y}\right) u+a u=0, \\
\operatorname{supp} a \subset\{(t, x, y) \in \Omega ; t \geq 0\}=\operatorname{supp} u, \\
\left|D^{\nu} u\right|+\left|D^{\nu} a\right| \leq C^{\nu+1}\left(\nu L_{\nu}\right)^{\nu}, \quad \nu=0,1, \ldots,
\end{gathered}
$$

where $D^{\nu}$ denotes any partial derivative of order $\nu$ and $0^{0}=1$.

With $L_{\nu}=(\log (\nu+2))^{\gamma}$ where $\gamma>2$ we conclude that $u$ and $a$ are contained in every Gevrey class $G_{\varrho}$ with $\varrho>1$, for it corresponds to $L_{\nu}=\nu^{\varrho-1}$. (An even better class is obtained by taking $L_{\nu}=(\log (\nu+2))^{2}(\log \log (\nu+4))^{\gamma}$ with $\gamma>2$, and so on with iterated logarithms.) Without the square root (1) would be the condition for nonquasianalyticity. We do not know if the stronger condition (1) is essential.

Proof of Theorem 1. For arbitrary $\beta$ and $\gamma \neq 0$, the differential equation (2) with $a=0$ is satisfied by

$$
u(t, x, y)=(1-i \beta y / \gamma)^{-\frac{1}{2}} \exp \left(i x / \beta-\frac{1}{2} t^{2} /(\gamma-i \beta y)\right)
$$

Received June 27, 2000. 
and we also have $D_{x} u=u / \beta, 2 D_{y} u=\beta D_{t}^{2} u$. Thus (5) is obtained from the standard fundamental solution of the heat (or Schrödinger) equation, and we have

$$
D_{x}^{l} D_{y}^{m} D_{t}^{n} u=2^{-m} \beta^{m-l} D_{t}^{2 m+n} u .
$$

To estimate the derivatives of $u$ we shall use the inequality

$$
\left|D_{t}^{n} \exp \left(-t^{2} / 2 c\right)\right| \leq 3(n / \operatorname{Re} c)^{n / 2}, \quad t \in \mathbf{R}, \operatorname{Re} c>0 .
$$

It follows from Cauchy's inequalities, for if $b=1 / 2 c$ and $z \in \mathbf{C},|z| \leq R$, then $2 \operatorname{Re} c=\operatorname{Re} b /|b|^{2}$ and

$$
\begin{aligned}
\left|\exp \left(-b(t+z)^{2}\right)\right| \leq \exp \left(-t^{2} \operatorname{Re} b\right. & \left.+2|b||t| R+|b| R^{2}\right) \\
& \leq \exp \left(\left(|b|+|b|^{2} / \operatorname{Re} b\right) R^{2}\right) \leq \exp \left(R^{2} / \operatorname{Re} c\right)
\end{aligned}
$$

hence

$$
\left|D_{t}^{n} \exp \left(-t^{2} / 2 c\right)\right| \leq n ! \min _{R>0} R^{-n} \exp \left(R^{2} / \operatorname{Re} c\right)=n !(e / \operatorname{Re} c)^{n / 2}(n / 2)^{-n / 2},
$$

which implies (7). Thus we have by (6) and (7), if $u$ is defined by (5) with $\beta>0$ and $\gamma>0$,

$$
\left|D_{x}^{l} D_{y}^{m} D_{t}^{n} u\right| \leq 2^{2-m}(2 / \gamma)^{m+\frac{1}{2} n} \beta^{m-l}\left(m+\frac{1}{2} n\right)^{m+\frac{1}{2} n} .
$$

Let $\Phi$ be a positive $C^{1}$ convex function on $\mathbf{R}_{+}$such that

$$
\Phi(k) \downarrow 0, \quad \Phi^{\prime}(k+1) / \Phi^{\prime}(k) \rightarrow 1 \quad \text { as } k \rightarrow \infty .
$$

(At the end of the proof such a function will be constructed from the sequence $\left(L_{\nu}\right)$ in the theorem.) If $\beta$ and $\gamma$ are replaced by $M^{-1} e^{-k} \Phi^{\prime}(k)^{2}$ with a large positive constant $M$ to be chosen later, it follows after a translation in $t$ that

$$
u_{k}(t, x, y)=\frac{\exp \left(-3 e^{k}\right)}{\sqrt{1-i y}} \exp \left(\frac{M e^{k}}{\Phi^{\prime}(k)^{2}}\left(i x-\frac{(t-\Phi(k))^{2}}{2(1-i y)}\right)\right)
$$

also satisfies (2) with $a=0$, and $u_{k}$ is concentrated at the plane where $t=\Phi(k)$. It is convenient to introduce a notation for a normalised $t$ variable centered there,

$$
t_{k}=-(t-\Phi(k)) / \Phi^{\prime}(k) .
$$

We have

$$
\begin{aligned}
t_{k \pm 1} & =-(t-\Phi(k \pm 1)) / \Phi^{\prime}(k \pm 1) \\
& =-\left(-t_{k} \Phi^{\prime}(k)+\Phi(k)-\Phi(k \pm 1)\right) / \Phi^{\prime}(k \pm 1) \\
& =t_{k} \Phi^{\prime}(k) / \Phi^{\prime}(k \pm 1)+(\Phi(k \pm 1)-\Phi(k)) / \Phi^{\prime}(k \pm 1) \\
& =t_{k} \pm 1+o(1), \quad \text { as } k \rightarrow+\infty,\left|t_{k}\right| \leq 1
\end{aligned}
$$


for $\Phi^{\prime}(k-1) \leq \Phi^{\prime}(\kappa) \leq \Phi^{\prime}(k+1)<0$ when $|\kappa-k|<1$, and $\Phi^{\prime}(k-1) / \Phi^{\prime}(k+1) \rightarrow 1$ as $k \rightarrow+\infty$. With some large positive integer $k_{0}$ to be chosen later we set

$$
u(t, x, y)= \begin{cases}\sum_{k=k_{0}}^{\infty} \chi\left(t_{k}\right) u_{k}(t, x, y), & \text { if } t \leq \Phi\left(k_{0}\right) \\ u_{k_{0}}(t, x, y), & \text { if } t>\Phi\left(k_{0}\right)\end{cases}
$$

Here $\chi \in C_{0}^{\infty}\left(\left(-\frac{4}{5}, \frac{4}{5}\right)\right)$ is equal to 1 in $\left[-\frac{3}{4}, \frac{3}{4}\right]$ and

$$
\left.\left|\chi^{(n)}(t) \leq C^{n} n^{n}\right| \Phi^{\prime}(\log (n+2))\right|^{-n}, n=0,2, \ldots
$$

Such a function exists by the Denjoy-Carleman theorem, for $\sum_{1}^{\infty}\left|\Phi^{\prime}(\log n)\right| / n$ is finite since

$$
\int_{c}^{\infty} t^{-1}\left|\Phi^{\prime}(\log t)\right| d t=\int_{\log c}^{\infty}-\Phi^{\prime}(s) d s=\Phi(\log c)
$$

and $t /\left|\Phi^{\prime}(\log t)\right|$ is increasing. By (14) we have

$$
\left|\frac{d^{n}}{d t^{n}} \chi\left(t_{k}\right)\right| \exp \left(-e^{k}\right) \leq C^{n} n^{n}\left|\Phi^{\prime}(\log (n+2))\right|^{-n}\left|\Phi^{\prime}(k)\right|^{-n} \exp \left(-e^{k}\right) .
$$

To estimate the product of the last two factors we note that since we have $\Phi^{\prime}(s) / \Phi^{\prime}(s+1) \rightarrow 1$ as $s \rightarrow \infty$, it follows for every $\varepsilon>0$ that $\left|\Phi^{\prime}(s)\right| e^{\varepsilon s} \rightarrow \infty$ as $s \rightarrow \infty$, so $\left|\Phi^{\prime}(k)\right|^{-n} \exp \left(-e^{k}\right) \rightarrow 0$ as $k \rightarrow \infty$. If $k>0$ when the maximum with respect to $k$ is attained then

$$
\begin{aligned}
& \left|\Phi^{\prime}(k-1)\right|^{-n} \exp \left(-e^{k-1}\right) \leq\left|\Phi^{\prime}(k)\right|^{-n} \exp \left(-e^{k}\right), \\
& \text { thus } \exp \left(e^{k}\left(1-e^{-1}\right)\right) \leq\left(\Phi^{\prime}(k-1) / \Phi^{\prime}(k)\right)^{n} .
\end{aligned}
$$

This implies that $e^{k}<n$ if $k>k_{1}$ and $k_{1}$ is large enough, so we obtain

$$
\left|\Phi^{\prime}(k)\right|^{-n} \exp \left(-e^{k}\right) \leq\left|\Phi^{\prime}\left(\max \left(\log n, k_{1}\right)\right)\right|^{-n}, \quad n=0,1, \ldots,
$$

hence with a new constant $C$

$$
\left|\frac{d^{n}}{d t^{n}} \chi\left(t_{k}\right)\right| \leq \exp \left(e^{k}\right) C^{n} n^{n}\left|\Phi^{\prime}(\log (n+2))\right|^{-2 n}, \quad n=0,1, \ldots
$$

With $\nu=l+m+n$ we have by (8) and (16)

$$
\begin{gathered}
\left|D_{x}^{l} D_{y}^{m} D_{t}^{n} u_{k}\right| \exp \left(e^{k}\right) \leq 2^{2+\frac{1}{2} n}\left(M e^{k} \Phi^{\prime}(k)^{-2}\right)^{l+\frac{1}{2} n}\left(m+\frac{1}{2} n\right)^{m+\frac{1}{2} n} \exp \left(-2 e^{k}\right) \\
\leq 2^{2+\frac{1}{2} n} M^{l+\frac{1}{2} n}\left(l+\frac{1}{2} n\right)^{l+\frac{1}{2} n}\left|\Phi^{\prime}(\log (2 l+n+2))\right|^{-2 l-n}\left(m+\frac{1}{2} n\right)^{m+\frac{1}{2} n} \\
\leq C^{\nu+1} \nu^{\nu}\left|\Phi^{\prime}(\log (\nu+2))\right|^{-2 \nu}
\end{gathered}
$$


where $C$ depends on $M$. We have also used that $e^{k \mu} \exp \left(-e^{k}\right)<\mu^{\mu}$ if $\mu>0$, with $\mu=l+\frac{1}{2} n$, and that $\Phi^{\prime}(\log (\nu+2)) / \Phi^{\prime}(\log (2 \nu+2))$ is bounded. Combined with $(15)^{\prime}$ this proves that with another constant $C$ depending on $M$

$$
\left|D_{x}^{l} D_{y}^{m} D_{t}^{n}\left(\chi\left(t_{k}\right) u_{k}(t, x, y)\right)\right| \leq C^{\nu+1} \nu^{\nu} \Phi^{\prime}(\log (\nu+2))^{-2 \nu}, \quad l+m+n \leq \nu .
$$

For $u$ we have the same bound apart from a factor 2 , for at most two supports of the terms in the series (13) can overlap if $k_{0}$ is large enough.

For the $k$ th term in (13) we have

$$
\begin{aligned}
-\left(D_{t}^{2}-2 D_{x} D_{y}\right) & \left(\chi\left(t_{k}\right) u_{k}(t, x, y)\right) \\
& =\Phi^{\prime}(k)^{-2} \chi^{\prime \prime}\left(t_{k}\right) u_{k}(t, x, y)-2 i \Phi^{\prime}(k)^{-1} \chi^{\prime}\left(t_{k}\right) D_{t} u_{k}(t, x, y) .
\end{aligned}
$$

Thus $t_{k} \in\left[-\frac{4}{5},-\frac{3}{4}\right] \cup\left[\frac{3}{4}, \frac{4}{5}\right]$ in the support, and it follows from (12) that

$$
\frac{1}{5}+o(1) \leq \pm t_{k \pm 1} \leq \frac{1}{4}+o(1) \quad \text { if } \mp t_{k} \in\left[\frac{3}{4}, \frac{4}{5}\right] .
$$

Hence $t_{k}^{2} \geq \frac{9}{16}$ and $t_{k \pm 1}^{2} \leq \frac{1}{15}$ then if $k$ is large enough. This means that if we set

$$
q_{k}(t, x, y)=u_{k}(t, x, y) / u_{k \pm 1}(t, x, y) \quad \text { when } \mp t_{k} \in\left[\frac{3}{4}, \frac{4}{5}\right]
$$

then for large $k$

$$
\left|q_{k}(t, x, y)\right| \leq \exp \left(e^{k}((M e / 30+3 e)-9 M / 64-3)\right), \quad \mp t_{k} \in\left[\frac{3}{4}, \frac{4}{5}\right]
$$

We choose $M$ now so that $M\left(\frac{e}{30}-\frac{9}{64}\right)+3 e<-1$ and conclude that

$$
\left|q_{k}(t, x, y)\right| \leq \exp \left(-4 e^{k}\right), \quad \text { if }\left|t_{k}\right| \in\left[\frac{3}{4}, \frac{4}{5}\right]
$$

To satisfy the equation (2) we must define in these intervals, except the one to the right of $\Phi\left(k_{0}\right)$,

$$
a(t, x, y)=\left(\frac{\chi^{\prime \prime}\left(t_{k}\right)}{\Phi^{\prime}(k)^{2}}-\frac{2 i \chi^{\prime}\left(t_{k}\right)}{\Phi^{\prime}(k)} D_{t} \log u_{k}(t, x, y)\right) \frac{q_{k}(t, x, y)}{1+\chi\left(t_{k}\right) q_{k}(t, x, y)}
$$

Outside all such intervals we define $a=0$. That $a$ is then in $C^{\infty}$ follows at once from (18), for differentiation of (19) can only lead to a loss of powers of $e^{k}$ and $\Phi^{\prime}(k)$ in the estimates. However, to prove the theorem we must give precise estimates for the derivatives.

The estimate (18) remains valid for $\left(y, t_{k}\right)$ in a complex $\mathbf{C}^{2}$ neighborhood of $E=\left\{(y, s) \in \mathbf{R}^{2} ;|y| \leq 1,|s| \in\left[\frac{3}{4}, \frac{4}{5}\right]\right\}$ which is independent of $k$ for large $k$. 
COUNTEREXAMPLE TO THE UNIQUENESS OF THE CAUCHY PROBLEM 619

Since $\partial / \partial t=-\Phi^{\prime}(k)^{-1} \partial / \partial t_{k}$, it follows using (16) as in the estimates following $(15)^{\prime}$ that for $\left(y, t_{k}\right) \in E$ and $l+m+n \leq \nu$

$$
\begin{array}{r}
\left|D_{x}^{l} D_{y}^{m} D_{t}^{n} q_{k}(t, x, y)\right| \leq C_{1}^{\nu+1}\left(e^{k} \Phi^{\prime}(k)^{-2}\right)^{l}(m+n) !\left|\Phi^{\prime}(k)\right|^{-n} \exp \left(-4 e^{k}\right) \\
\leq C_{2}^{\nu+1} l^{l}(m+n)^{m+n}\left|\Phi^{\prime}(\log (2 l+n+2))\right|^{-2 l-n} \exp \left(-2 e^{k}\right) \\
\leq C_{3}^{\nu+1} \nu^{\nu} \Phi^{\prime}(\log (\nu+2))^{-2 \nu} \exp \left(-2 e^{k}\right)
\end{array}
$$

if $k$ is large enough. Here $C_{1}, C_{2}, C_{3}$ depend on $M$. Using $(15)^{\prime}$ we conclude that

$$
\left|D_{x}^{l} D_{y}^{m} D_{t}^{n}\left(\chi\left(t_{k}\right) q_{k}(t, x, y)\right)\right| \leq C_{4}^{\nu+1} \nu^{\nu} \Phi^{\prime}(\log (\nu+2))^{-2 \nu} \exp \left(-e^{k}\right) .
$$

By the results on inverse closed Denjoy-Carleman classes of Rudin [5, Theorem A], an estimate such as (20) follows for $q_{k}(t, x, y) /\left(1+\chi\left(t_{k}\right) q_{k}(t, x, y)\right)$ since $\left|\Phi^{\prime}\right|^{-1}$ is increasing. In (19) we have

$$
D_{t} \log u_{k}(t, x, y)=-i M e^{k} \Phi^{\prime}(k)^{-1} t_{k} /(1-i y)
$$

and

$$
\begin{aligned}
\mid D_{y}^{m} D_{t}^{n}\left(\Phi^{\prime}(k)^{-2} \chi^{\prime \prime}\left(t_{k}\right)-\right. & \left.2 \Phi^{\prime}(k)^{-2} M e^{k} \chi^{\prime}\left(t_{k}\right) t_{k} /(1-i y)\right) \mid \exp \left(-2 e^{k}\right) \\
& \leq C_{5}^{m+n+1} m ! n^{n}\left|\Phi^{\prime}(\log (n+2))\right|^{-2(n+2)} \\
& \leq C_{6}^{n+m+1}(n+m)^{n+m}\left|\Phi^{\prime}(\log (n+m+2))\right|^{-2(n+m)} .
\end{aligned}
$$

Hence we conclude that

$$
\left|D^{\nu} u(t, x, y)\right|+\left|D^{\nu} a(t, x, y)\right| \leq C^{\nu+1} \nu^{\nu} \Phi^{\prime}(\log (\nu+2))^{-2 \nu} .
$$

To complete the proof of Theorem 1 we must choose an appropriate function $\Phi$ starting from the sequence $\left(L_{\nu}\right)$ in the theorem. At first we assume that $L_{2 \nu} / L_{\nu} \rightarrow 1$ as $\nu \rightarrow \infty$, interpolate $L_{\nu}$ linearly between the integers and define, at first for large positive $t$,

$$
\varphi(t)=L_{e^{t}}^{-\frac{1}{2}} .
$$

Then $\varphi$ is decreasing and $\varphi(t+1) \geq L_{4 e^{-\frac{1}{2}}}$, so $\varphi(t+1) / \varphi(t) \rightarrow 1$ as $t \rightarrow \infty$. By (1) we have

$$
\int_{t_{0}}^{\infty} \varphi(t) d t=\int_{\exp \left(t_{0}\right)}^{\infty} L_{s}^{-\frac{1}{2}} d s / s<\infty .
$$

If we define $\Phi(k)=\int_{k}^{\infty} \varphi(t) d t$ then

$$
\Phi^{\prime}(k)=-\varphi(k)=-L_{e^{k}}^{-\frac{1}{2}}, \quad \Phi^{\prime}(\log \nu)^{-2}=L_{\nu},
$$

so (4) follows from (21). 
To eliminate the hypothesis that $L_{2 \nu} / L_{\nu} \rightarrow 1$ as $\nu \rightarrow \infty$ we observe that if $a_{\nu}=\left(L_{2^{\nu}}\right)^{-\frac{1}{2}}$ then $a_{\nu}$ is decreasing and $\sum_{1}^{\infty} a_{\nu}<\infty$ since

$$
\sum_{2^{\nu-1}<n \leq 2^{\nu}} \frac{1}{n \sqrt{L_{n}}} \geq a_{\nu} / 2, \quad \nu=1,2, \ldots
$$

By Lemma 2 below we can choose a decreasing sequence $A_{\nu} \geq a_{\nu}$ such that $\sum A_{\nu}<\infty$ and $A_{\nu+1} / A_{\nu} \rightarrow 1$ as $\nu \rightarrow \infty$. If we define $\tilde{L}_{n}=A_{\nu}^{-2}$ when $2^{\nu-1}<n \leq 2^{\nu}$ then $\tilde{L}_{n} \leq a_{\nu}^{-2} \leq L_{n}$ for these indices, $\tilde{L}_{n}$ is increasing, (1) is valid with $L$ replaced by $\bar{L}$, and $\tilde{L}_{2 n} / \tilde{L}_{n} \rightarrow 1$ as $n \rightarrow \infty$. Thus we have proved that $u$ and $a$ can be chosen so that (2), (3), (4) are valid with $L$ replaced by $\tilde{L}$, which implies that (4) holds for the original sequence $L$. The proof is completed by the following lemma.

Lemma 2. Let $\left(a_{\nu}\right)_{0}^{\infty}$ be a positive decreasing sequence such that $\sum_{0}^{\infty} a_{\nu}<\infty$. Then there exists another positive decreasing sequence $\left(A_{\nu}\right)_{0}^{\infty}$ such that

$$
A_{\nu} \geq a_{\nu}, \nu=0,1, \ldots, \quad \sum_{0}^{\infty} A_{\nu}<\infty, \lim _{\nu \rightarrow \infty} A_{\nu+1} / A_{\nu}=1 .
$$

Proof. If $a_{\nu}$ is exponentially decreasing we can just take $A_{\nu}=C(\nu+1)^{-2}$ with $C=\sup a_{\nu}(\nu+1)^{2}$, so we may assume that $a_{\nu}$ is not exponentially decreasing. As a first step we shall prove that if $t \in(0,1)$ then

$$
A_{\nu}=\max _{0 \leq \mu \leq \nu} a_{\mu} t^{\nu-\mu}
$$

is a decreasing sequence with

$$
A_{\nu} \geq a_{\nu}, A_{\nu+1} \geq t A_{\nu}, \nu=0,1, \ldots ; \quad \sum_{A_{\nu}>a_{\nu}} A_{\nu} \leq a_{0} t /(1-t) .
$$

The first two inequalities are obvious. If $A_{\nu}>a_{\nu}$ then $A_{\nu}=t A_{\nu-1} \leq A_{\nu-1}$, and if $A_{\nu}=a_{\nu}$ then $A_{\nu} \leq a_{\nu-1} \leq A_{\nu-1}$ so the sequence is decreasing. Moreover, if $\mathcal{O}=\left\{\nu ; A_{\nu}>a_{\nu}\right\}$ then $A_{\nu} \leq A_{0} t^{\kappa+1}=a_{0} t^{\kappa+1}$ if $\nu \in \mathcal{O}$ and there are $\kappa$ elements in $\mathcal{O}$ which are smaller than $\nu$, which proves the last inequality in (23).

Set $t_{k}=k /(k+1)$ and choose an increasing sequence $\nu_{k}$ of positive integers such that

$$
a_{\nu_{k}} t_{k}^{-\nu_{k}}>a_{\mu} t_{k}^{-\mu} \quad \text { when } \mu<\nu_{k} ; \quad \sum_{1}^{\infty} k a_{\nu_{k}}<\infty .
$$

This is possible since $a_{\nu} t_{k}^{-\nu}$ is not bounded when $\nu \rightarrow \infty$ for fixed $k$. Set $\nu_{0}=0$ and

$$
A_{\nu}=\max _{0 \leq \mu \leq \nu} a_{\mu} t_{k}^{\nu-\mu}=\max _{\nu_{k} \leq \mu \leq \nu} a_{\mu} t_{k}^{\nu-\mu} \quad \text { when } \nu_{k} \leq \nu \leq \nu_{k+1}, \quad k=0,1,2, \ldots
$$


The choice of $\nu_{k}$ gives $A_{\nu_{k}}=a_{\nu_{k}}$ and $A_{\nu_{k+1}}=a_{\nu_{k+1}}$ so the definitions agree at the end points, $A_{\nu}$ is a decreasing sequence $\geq a_{\nu}$, such that $A_{\nu+1} \geq t_{k} A_{\nu}$ when $\nu \geq \nu_{k}, k \geq 1$, and

$$
\sum_{0}^{\infty} A_{\nu} \leq \sum_{0}^{\infty} a_{\nu}+\sum_{1}^{\infty} a_{\nu_{k}} t_{k} /\left(1-t_{k}\right)=\sum_{0}^{\infty} a_{\nu}+\sum_{1}^{\infty} k a_{\nu_{k}}<\infty
$$

which completes the proof. ${ }^{1}$

A rotation of the $x, y$ coordinates replaces the equation (2) by

$$
\left(D_{t}^{2}+D_{x}^{2}-D_{y}^{2}\right) u+a u=0
$$

which is hyperbolic with respect to $y$. We can also replace $u$ and $a$ by $u(\varepsilon t, \varepsilon x, \varepsilon y)$ and $\varepsilon^{2} a(\varepsilon t, \varepsilon x, \varepsilon y)$ to make $a$ and $u$ defined in any desired bounded open set containing the origin, and at the same time make the constant $C$ in (4) as small as we wish. To discuss a counterexample due to Métivier [4] we also need another solution of the equation $(2)^{\prime}$ which is different from 0 in $\Omega$, given by the following proposition:

Proposition 3. Let $\left(L_{\nu}\right)_{0}^{\infty}$ be any positive increasing sequence with $L_{0}=1$ and let $a \in C^{\infty}(\Omega)$ where $\Omega=\left\{(t, x, y) \in \mathbf{R}^{3} ;|y|+\sqrt{t^{2}+x^{2}}<1\right\}$. If

$$
\left|D^{\nu} a(t, x, y)\right| \leq C_{a}^{\nu+1}\left(\nu L_{\nu}\right)^{\nu}, \quad(t, x, y) \in \Omega, \nu=0,1, \ldots,
$$

where $C_{a}<\frac{1}{4}$, then the Cauchy problem

$$
\left(D_{t}^{2}+D_{x}^{2}-D_{y}^{2}\right) v+a v=0 \text { in } \Omega, \quad v=1 \text { and } D_{y} v=0 \text { when } y=0
$$

has a unique solution in $C^{\infty}(\Omega)$, and

$$
\left|D^{\nu} v(t, x, y)\right| \leq C 2^{\nu}\left((\nu+2) L_{\nu+2}\right)^{\nu+2}, \quad(t, x, y) \in \Omega,|y|<\frac{1}{2}, \nu=0,1, \ldots
$$

Thus $|v(t, x, y)|>\frac{1}{2}$ if $54 C L_{3}^{3}|y|<1$ and $|y|<\frac{1}{2}$.

Proof. It is very well known that there is a unique $C^{\infty}$ solution so we shall just prove $(26)$ when $0 \leq y<\frac{1}{2}$ using that by the standard energy estimates, if $\left(D_{t}^{2}+D_{x}^{2}-D_{y}^{2}\right) u=f$ in $\Omega$ and

$$
E(y)=\left(\int_{(t, x, y) \in \Omega}\left(\left|D_{t} u(t, x, y)\right|^{2}+\left|D_{x} u(t, x, y)\right|^{2}+\left|D_{y} u(t, x, y)\right|^{2}\right) d t d x\right)^{\frac{1}{2}}
$$

\footnotetext{
${ }^{1}$ The author is grateful to Jan Boman for an observation which simplified the proof.
} 
is the energy of $u$ at "time" $y$, then

$$
\begin{aligned}
E^{\prime}(y) & \leq\left(\int_{(t, x, y) \in \Omega}|f(t, x, y)|^{2} d t d x\right)^{\frac{1}{2}}=\|f(\cdot, \cdot, y)\|, \quad 0 \leq y<1, \\
E(y) & \leq E(0)+\int_{0}^{y}\|f(\cdot, \cdot, s)\| d s, \quad 0 \leq y<1, \\
\|u(\cdot, \cdot, y)\| & \leq\|u(\cdot, \cdot, 0)\|+\int_{0}^{y} E(s) d s, \quad 0 \leq y<1 .
\end{aligned}
$$

For the solution $v$ of the Cauchy problem (25) we have

$$
E^{\prime}(y) \leq C_{a}\|v(\cdot, \cdot, y)\| \leq C_{a}\left(2+\int_{0}^{y} E(s) d s\right), 0 \leq y<1, \quad E(0)=0,
$$

which implies that $E(y) \leq 2 \sqrt{C_{a}} \sinh \left(\sqrt{C_{a}} y\right)<1$, and that $\|v(\cdot, \cdot, y)\| \leq$ $2 \cosh \left(\sqrt{C_{a}} y\right)<3$. We want to prove inductively that the energy of $v_{l, n}=$ $D_{x}^{l} D_{t}^{n} v$ is $\leq\left(\nu L_{\nu}\right)^{\nu}$ if $l+n=\nu$. When $\nu \neq 0$ the Cauchy data of $v_{l, n}$ are equal to 0 , and if the claim is proved for smaller values of $\nu$, hence $\left\|D_{x}^{l} D_{t}^{n} v\right\| \leq$ $\left((l+n-1) L_{l+n-1}\right)^{l+n-1}$ when $0<l+n \leq \nu$, we have by $(24)$ when $l+n=\nu$

$$
\begin{aligned}
\left\|D_{x}^{l} D_{t}^{n}(a v)(\cdot, \cdot, y)\right\| \\
\quad \leq \sum_{j=0}^{\nu-1}\left(\begin{array}{c}
\nu \\
j
\end{array}\right) C_{a}^{j+1}\left(j L_{j}\right)^{j}\left((\nu-1-j) L_{\nu-1-j}\right)^{\nu-1-j}+3 C_{a}^{\nu+1}\left(\nu L_{\nu}\right)^{\nu} \\
\quad \leq \frac{1}{4} \sum_{j=0}^{\nu-1}\left(\begin{array}{c}
\nu-1 \\
j
\end{array}\right)\left(j L_{j}\right)^{j}\left((\nu-1-j) L_{\nu-1-j}\right)^{\nu-1-j}+\frac{3}{16}\left(\nu L_{\nu}\right)^{\nu} \\
\leq \frac{1}{4} L_{\nu}^{\nu} \sum_{j=0}^{\nu-1}\left(\begin{array}{c}
\nu-1 \\
j
\end{array}\right) j^{j}(\nu-1-j)^{\nu-1-j}+\frac{3}{16}\left(\nu L_{\nu}\right)^{\nu} \leq\left(\nu L_{\nu}\right)^{\nu},
\end{aligned}
$$

where we have used the inequalities

$$
\begin{gathered}
\left(\begin{array}{c}
\nu \\
j
\end{array}\right) C_{a}^{j+1} /\left(\begin{array}{c}
\nu-1 \\
j
\end{array}\right)=C_{a}^{j+1} \nu /(\nu-j) \leq C_{a}^{j+1}(j+1) \leq \frac{1}{4}, \\
\sum_{j=0}^{\mu}\left(\begin{array}{c}
\mu \\
j
\end{array}\right) j^{j}(\mu-j)^{\mu-j} \leq 2 \mu^{\mu+\frac{1}{2}}
\end{gathered}
$$

the second follows easily from Stirling's formula. Hence the energy of $v_{l, n}$ is $\leq\left(\nu L_{\nu}\right)^{\nu}$.

We have now proved that

$$
\left\|D_{x}^{l} D_{y}^{m} D_{t}^{n} v(\cdot, \cdot, y)\right\| \leq\left(\nu L_{\nu}\right)^{\nu}, \quad \text { if } 0 \neq \nu=l+m+n, m \leq 1
$$

and we want to conclude that

$$
\left\|D_{x}^{l} D_{y}^{m} D_{t}^{n} v(\cdot, \cdot, y)\right\| \leq 2^{m}\left(\nu L_{\nu}\right)^{\nu}, \quad \text { if } 0 \neq \nu=l+m+n
$$


also when $m \geq 2$. Assuming this proved already for smaller values of $m$ we have since $D_{x}^{l} D_{y}^{m} D_{t}^{n} v=D_{x}^{l} D_{y}^{m-2} D_{t}^{n}\left(D_{x}^{2} v+D_{t}^{2} v+a v\right)$

$$
\begin{aligned}
&\left\|D_{x}^{l} D_{y}^{m} D_{t}^{n} v(\cdot, \cdot, y)\right\| \leq 2^{m-1}\left(\nu L_{\nu}\right)^{\nu} \\
&+2^{m-2} \sum_{j=0}^{\nu-2}\left(\begin{array}{c}
\nu-2 \\
j
\end{array}\right) C_{a}^{j+1}\left(j L_{j}\right)^{j}\left((\nu-2-j) L_{\nu-2-j}\right)^{\nu-2-j}+3 C_{a}^{\nu-1}\left(\nu L_{\nu}\right)^{\nu} \\
& \leq 2^{m-1}\left(\nu L_{\nu}\right)^{\nu}+2^{m-2} \frac{1}{4} L_{\nu}^{\nu} 2 \nu^{\nu}+\frac{3}{4}\left(\nu L_{\nu}\right)^{\nu} \\
& \leq 2^{m-1}\left(\nu L_{\nu}\right)^{\nu}\left(1+\frac{1}{4}+\frac{3}{8}\right)<2^{m}\left(\nu L_{\nu}\right)^{\nu}
\end{aligned}
$$

which proves (27). An application of Sobolev's inequality in the $t, x$ variables gives (26).

We shall now recall an example of nonuniqueness for the nonlinear Cauchy problem due to Métivier [4]. Let $\left(L_{\nu}\right)$ be a positive increasing sequence satisfying (1). By using first Theorem 1 and then Proposition 3 we can find functions $u$, $a$, and $v$ in the unit ball $\Omega$ in $\mathbf{R}^{3}$ satisfying (4) such that

$$
L u+a u=L v+a v=0 \quad \text { in } \Omega,
$$

where $L=\partial_{t}^{2}+\partial_{x}^{2}-\partial_{y}^{2}$, and

$$
\operatorname{supp} u=\{(t, x, y) \in \Omega ; t \geq 0\}, \quad|u|<1<|v| \text { in } \Omega .
$$

Choose $\chi \in C^{\infty}(\mathbf{R})$ so that estimates of the form (4) are valid for $\chi$ and $\sup |\chi| \leq$ $1, \chi(z)=0$ when $z \leq 0$. If

$$
u_{\chi}(t, x, y, z)=v(t-z, x, y)+\chi(z) u(t-z, x, y)
$$

it follows that in $\widetilde{\Omega}=\left\{(t, x, y, z) \in \mathbf{R}^{4} ;(t-z, x, y) \in \Omega\right\}$

$$
\left|D^{\nu} u_{\chi}(t, x, y, z)\right| \leq C^{\nu+1}\left(\nu L_{\nu}\right)^{\nu}, \quad \nu=0,1, \ldots,
$$

and $(L+b) u_{\chi}=0$ if $b(t, x, y, z)=a(t-z, x, y)$, for $L$ does not act on $\chi$. Since $u_{\chi} \neq 0$ in $\widetilde{\Omega}$ we can define $v_{\chi}(t, x, y, z)=\log u_{\chi}(t, x, y, z)$ uniquely so that $v_{\chi}(0,0,0,0)$ is a given value of $\log v(0,0,0)$. Since $L_{\nu}$ is increasing it follows again from Rudin [5] that $v_{\chi}$ also has bounds of the form (28), and the equation $(L+b) u_{\chi}=0$ means that

$$
L v_{\chi}+\left(\partial_{t} v_{\chi}\right)^{2}+\left(\partial_{x} v_{\chi}\right)^{2}-\left(\partial_{y} v_{\chi}\right)^{2}+b=0 .
$$

Since $\left(\partial_{t}+\partial_{z}\right) b=0$ it follows that $v_{\chi}$ is a solution of the equation

$$
\left(\partial_{t}+\partial_{z}\right)\left(\partial_{t}^{2} U+\partial_{x}^{2} U-\partial_{y}^{2} U+\left(\partial_{t} U\right)^{2}+\left(\partial_{x} U\right)^{2}-\left(\partial_{y} U\right)^{2}\right)=0
$$


for every $\chi$. We have $u_{\chi}-u_{0}=\chi(z) u(t-z, x, y)$, hence

$$
\begin{aligned}
& \operatorname{supp}\left(u_{\chi}-u_{0}\right) \subset\{(t, x, y, z) \in \widetilde{\Omega} ; t \geq z \geq 0\}, \\
& \operatorname{supp}\left(v_{\chi}-v_{0}\right) \subset\{(t, x, y, z) \in \widetilde{\Omega} ; t \geq 0\},
\end{aligned}
$$

and $(0,0,0,0) \in \operatorname{supp}\left(u_{\chi}-u_{0}\right) \subset \operatorname{supp}\left(v_{\chi}-v_{0}\right)$ if $0 \in \operatorname{supp} \chi$. Thus the equation (29) has a continuum of solutions satisfying

$$
\left|D^{\nu} U\right| \leq C^{\nu+1}\left(\nu L_{\nu}\right)^{\nu}, \quad \nu=0,1, \ldots,
$$

which coincide on one side of the noncharacteristic plane where $t=0$ whereas no two are equal in a neighborhood of the origin. This is a special case of Proposition 3 in [4] apart from the regularity property (30). In particular, the solutions belong to every Gevrey class $G_{\varrho}$ with $\varrho>1$ if we choose $L_{\nu}=(\log (\nu+2))^{\gamma}$ with $\gamma>2$.

\section{References}

[1] S. Alinhac and M. S. Baouendi, A non uniqueness result for operators of principal type, Math. Z. 220 (1995), 561-568.

[2] $\longrightarrow$ A counterexample to strong uniqueness for partial differential equations of Schrödinger's type, Comm. Partial Differential Equations 19 (1994), 1727-1733.

[3] N. Lerner, Résultats d'unicité forte pour des opérateurs elliptiques à coefficients Gevrey, Comm. Partial Differential Equations 6 (1981), 1163-1177.

[4] G. Métivier, Counterexamples to Hölmgren's uniqueness for analytic nonlinear Cauchy problems, Invent. Math. 112 (1993), 217-222.

[5] W. Rudin, Division in algebras of infinitely differentiable functions, J. Math. Mech. 11 (1962), 797-809.

Department of Mathematics, University of Lund, Box 118, S-221 00 Lund, Sweden. E-mail address: lvh@maths.1th.se 